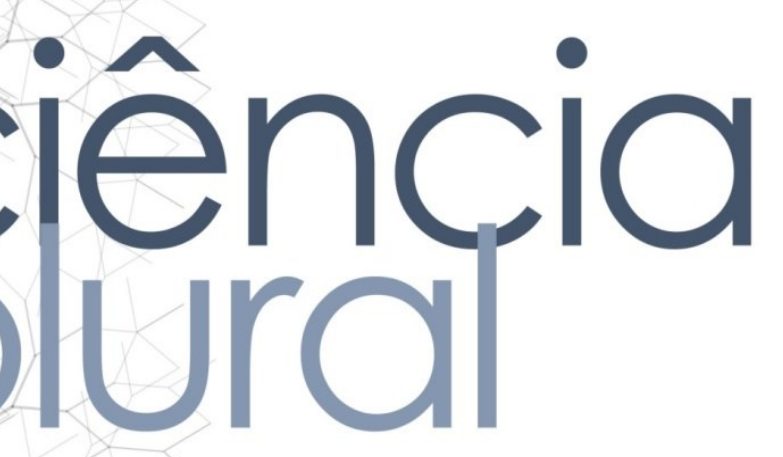

\title{
AVALIAÇÃO DAS CONSULTAS DE PRÉ-NATAL: ADESÃO DO PRÉ- NATAL E COMPLICAÇÕES NA SAÚDE MATERNO-INFANTIL
}

\section{Evaluation of prenatal consultations: prenatal access and complications in maternal-child health}

Dídia de Oliveira Pereira • Acadêmica do Curso de Enfermagem da Faculdade de Ciências da Saúde do Trairi (FACISA)/Universidade Federal do Rio Grande do Norte (UFRN). E-mail: didia_oliveira@hotmail.com

Tainara Lôrena dos Santos Ferreira • Acadêmica do Curso de Enfermagem da FACISA/UFRN. Bolsista de Iniciação Científica PIBIC CNPq-UFRN. E-mail: tainara_lorena@hotmail.com

Daísy Vieira de Araújo • Enfermeira. Mestre em Enfermagem. Professora da FACISA/UFRN.

Email:mestredaisy@yahoo.com.br

Káthya Daniella Figueiredo Melo • Secretaria Municipal de Saúde de Cuité, Paraíba.

Email: kathyadaniella@hotmail.com

Fábia Barbosa de Andrade • Enfermeira. Doutora em Ciências da Saúde. Professora Adjunto III da UFRN.

E-mail: fabiabarbosabr@yahoo.com.br

Autor responsável pela correspondência:

Dídia de Oliveira Pereira. E-mail: didia_oliveira@hotmail.com 


\section{ciência plural}

RESUMO

Introdução: As consultas de pré-natal realizadas na Atenção Primária à Saúde apresentam-se como momento indispensável para ofertar orientações necessárias no período da gestação e puerpério, sendo ocasião indispensável para que a grávida possa revelar seu medos, anseios e dúvidas sobre esse momento singular dela mesma e do feto. Objetivo: Avaliar a correlação entre adesão do pré-natal e complicações na saúde materno-infantil. Métodos: Trata-se de um estudo quantitativo e avaliativo dos indicadores de morbidade e mortalidade materno-infantil no município de Santa Cruz, no estado do Rio Grande do Norte, Brasil. Resultados: Das mães que aderiram ao pré-natal, $86 \%$ não complicaram, ao passo que $13 \%$ das grávidas tiveram complicação na gestação, tendo como causas sangramento, convulsão, infecção urinária, diabetes e hipertensão arterial. Conclusão: Destarte o pré-natal como importante para realizar um acompanhamento saudável da gestante e seu filho, prevenindo e/ou tratando as complicações que podem surgir nesse período, concorrendo, assim, para a diminuição dos índices de morbimortalidade materna e infantil.

Palavras-chaves: Cuidado Pré-Natal. Obstetrícia. Atenção Primária à Saúde.

\section{ABSTRACT}

Introduction: The prenatal consultations carried out in Primary Health Care are an indispensable moment to offer the necessary guidelines during pregnancy and puerperium, and it is an indispensable occasion for revealing their fears, anxieties and doubts about this singular moment of the woman and your fetus. Objective: To evaluate the correlation between prenatal adherence and maternal and child health complications. Methods: This is a quantitative and evaluative study of indicators of maternal and infant morbidity and mortality in the municipality of Santa Cruz, in the state of Rio Grande do Norte, Brazil. Results: A prevalence of $86 \%$ of the mothers who joined prenatal care did not complicate, whereas a prevalence of $13 \%$ was found to have complications during pregnancy, with bleeding, convulsion, urinary tract infection, diabetes and hypertension. Conclusion: To determine prenatal care as important for a healthy follow-up of the pregnant woman and her child, preventing and / or treating the complications that may arise during this period, thus contributing to the reduction of maternal and infant morbidity and mortality rates.

Keywords: Prenatal Care. Obstetrics. Primary Health Care. 


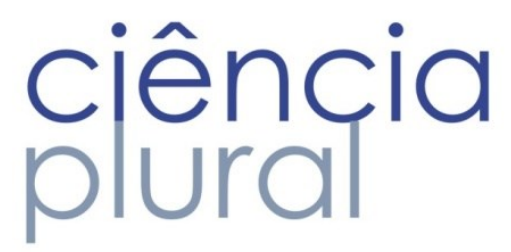

\section{Introdução}

A atividade de ser mãe é vista como uma das atribuições da mulher na sociedade, a qual tem durante esse período sua feminilidade aumentada, onde a principal necessidade são as orientações que devem ser realizadas na gestação e puerpério, repassadas durante as consultas de pré-natal na Atenção Primária à Saúde (APS).

Sendo assim, é essencial uma atenção de qualidade, onde os riscos gestacionais sejam identificados na anamnese e no exame clínico. Quando vista a necessidade, devem-se realizar medidas para amenizar os riscos de resultados indesejados, sempre orientando-as quanto à importância da prática de atividade física e da adesão à uma alimentação saudável1 ${ }^{1}$.

Desse modo, as gestantes têm em sua comunidade as consultas de pré-natal, como uma ocasião indispensável para que as mesmas e seus familiares possam revelar seu medos, anseios e dúvidas sobre esse momento singular da mulher e seu feto.

Em relação à taxa de mortalidade neonatal no Brasil, em 2007, era equivalente a 13,2/1000 nascidos vivos, e encontrava-se voltada a causas preveníveis, relacionadas ao acesso e utilização dos serviços de saúde, além da qualidade da assistência pré-natal, ao parto e ao recém-nascido. As afecções perinatais respondem atualmente por cerca de $60 \%$ das mortes infantis e $80 \%$ das mortes neonatais, além de serem a primeira causa de morte em menores de cinco anos ${ }^{2}$.

De tal forma, a disponibilidade e a qualidade da assistência que os serviços de saúde proporcionam às gestantes, recém-nascidos e crianças formam condicionantes indispensáveis à melhora das condições de saúde na infância, tais como, a relação entre ausência ou deficiência do pré-natal e o retardo do crescimento intrauterino, a prematuridade e, por extensão, a morbimortalidade infantil ${ }^{3}$.

Nesse sentido é necessário que haja uma conscientização maior por parte dos profissionais de saúde para com as gestantes, direcionando-as para uma gestação saudável ate o momento do parto e pós-parto, pois o sobrepeso ou obesidade materna, por exemplo, aumentam a probabilidade de possíveis complicações, tais como, diabetes gestacional, hipertensão na gravidez e parto prolongado.

Desse modo, esse estudo é de relevância para os profissionais da APS, para a gestão em saúde e comunidade, especialmente para gestantes e futuras genitoras, no intuito dos mesmos reconhecerem a 


\section{ciência plural}

importância do pré-natal e as prováveis complicações relacionadas à gestação. Assim, esse estudo tem o objetivo de avaliar a correlação entre adesão do pré-natal e complicações na saúde materno-infantil.

\section{Metodologia}

Trata-se de um estudo quantitativo e avaliativo dos indicadores de morbidade e mortalidade maternoinfantil nas Unidades Básicas de Saúde que integram a rede de Atenção Primária à Saúde (APS), no município de Santa Cruz, no estado do Rio Grande do Norte, Brasil.

A escolha da amostra se deu por amostragem sistemática com base no cadastro da rede de APS. A cidade é composta por 12 Equipes de Saúde da Família. A população foi constituída por mulheres mães de crianças menores de um ano (crianças com 11 meses e 29 dias). Por sua vez, a escolha da amostra se deu a partir do cálculo de uma prevalência de 19,3\% de mortalidade infantil em menores de ano no Estado do Rio Grande do Norte, adotando um erro permitido de 5\% e grau de confiança de $95 \%$ e um poder de $80 \%$, o que resultou em 200 mulheres, mães com filhos de menores de um ano e residentes na cidade de Santa Cruz e cadastradas na USF.

Para coleta de dados foi utilizado um instrumento validado pelo Ministério da Saúde e utilizado na Chamada Neonatal. As entrevistas foram realizadas no ano de 2013. Para análise dos dados foi utilizada a estatística descritiva por meio do conhecimento de frequências, medidas de tendência central e dispersão. Foi realizada ainda a análise bivariada e cálculo de teste de qui-quadrado e Odds Ratio (OR) com a utilização do Statistical Package for the Social Sciences (SPSS). O projeto foi submetido a avaliação ao comitê de Ética em Pesquisa da Faculdade de Ciências de Saúde do Trairí - UFRN e aprovado com número de parecer n 311.613.

\section{Resultados}

A média de idade das participantes foi em torno de 28,17 anos, mediana 27,0; e desvio padrão de 7,22. No tocante à escolaridade da mãe, a média em anos de estudos foi de 6,04 anos, mediana 7,0 e desvio padrão de 3,28

Ao analisar os dados, percebeu-se uma grande procura pelas gestantes em realizarem seu acompanhamento em um serviço público de saúde, comprovado por $75,4 \%$ ( $n=144)$ que realizaram na Unidade Básica de Saúde de sua área, 17,8\% ( $n=34)$ em hospital público de alto risco, 3,7\% ( $n=7)$ em consultório 


\section{ciência \\ plural}

particular, 1,6\% ( $n=3)$ em consultório ambulatorial com especialidade, 0,5\% $(n=1)$ na Unidade Básica de Saúde e no Hospital, $0,5 \%(n=1)$ em outro e $0,5 \%(n=1)$ não se aplica, revelando que a maioria dessas mulheres manifesta interesse no atendimento público que elas têm por direito, que é o pré-natal recomendado pelo Ministério da Saúde.

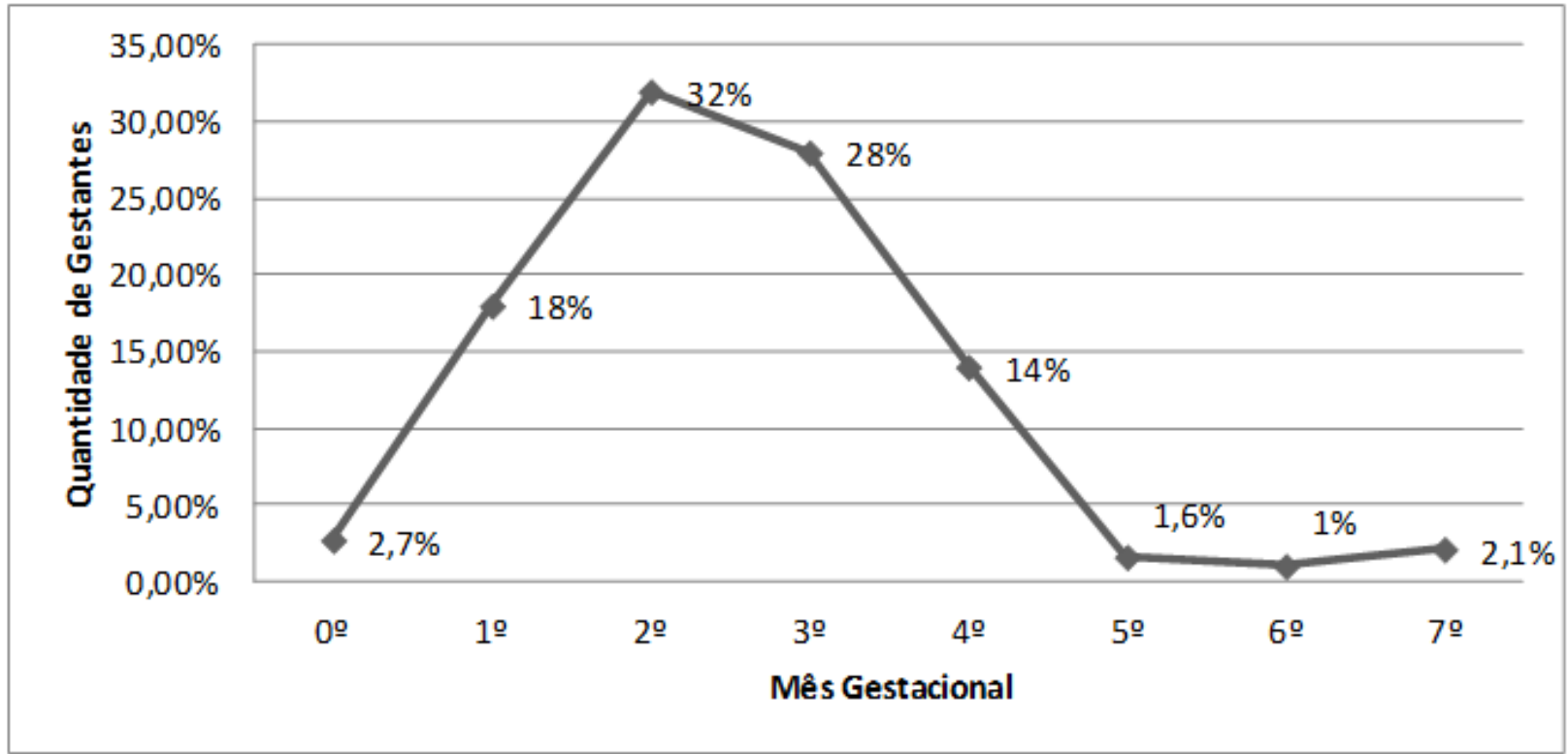

Gráfico 1: Distribuição do mês do início do Pré-natal na Atenção Primária à Saúde.

Santa Cruz/RN, Brasil, 2014

O Gráfico 1 mostra a partir de que mês gestacional as mulheres começaram a frequentar as consultas de pré-natal, revelando que $32 \%$ ( $n=61)$ iniciou no $2^{\circ}$ mês, $28 \%(n=54)$ começou no $3^{\circ}$ mês gestacional, no $1^{\circ}$ mês foram $18 \%(n=35)$, seguido de $14 \%(n=25)$ no $4^{\circ}$ mês, $2,7 \%(n=5)$ iniciaram antes de completar um mês de gravidez, $2,1 \%(n=4)$ no $7^{0}$ mês, no $5^{\circ}$ mês contabilizou 1,6\% $(n=3)$, no $6^{\circ}$ foi $1 \%(n=2)$ e $0,6 \%(n=1)$ que não se aplica, mostrando que a maioria das mulheres se interessam em procurar o serviço de saúde logo após terem a confirmação da gestação. 
Tabela 1: Classificação da correlação entre a realização do pré-natal e a qualidade do atendimento.

Santa Cruz/RN, Brasil, 2014.

\begin{tabular}{|c|c|c|c|c|c|c|c|c|}
\hline & \multicolumn{7}{|c|}{ QUALIDADE DE ATENDIMENTO } \\
\hline & & $\begin{array}{c}\text { Muito } \\
\text { Boa }\end{array}$ & Boa & Satisfatória & Ruim & $\begin{array}{l}\text { Muito } \\
\text { Ruim }\end{array}$ & $\begin{array}{l}\text { Não se } \\
\text { Aplica }\end{array}$ & Total \\
\hline \multirow{2}{*}{$\begin{array}{l}\text { PRÉ- } \\
\text { NATAL }\end{array}$} & Sim & $\begin{array}{c}26 \% \\
(n=52)\end{array}$ & $\begin{array}{c}59 \% \\
(n=118)\end{array}$ & $\begin{array}{c}8,5 \% \\
(n=17)\end{array}$ & $\begin{array}{l}1,0 \% \\
(n=2)\end{array}$ & $\begin{array}{l}1,0 \% \\
(n=2)\end{array}$ & $\begin{array}{c}0 \% \\
(n=0)\end{array}$ & $\begin{array}{c}95,5 \% \\
(n=191)\end{array}$ \\
\hline & Não & $\begin{array}{c}0 \% \\
(n=0)\end{array}$ & $\begin{array}{c}0 \% \\
(n=0)\end{array}$ & $\begin{array}{c}0 \% \\
(n=0)\end{array}$ & $\begin{array}{c}0 \% \\
(n=0)\end{array}$ & $\begin{array}{c}0 \% \\
(n=0)\end{array}$ & $\begin{array}{l}4,5 \% \\
(n=9)\end{array}$ & $\begin{array}{l}4,5 \% \\
(n=9)\end{array}$ \\
\hline \multicolumn{2}{|c|}{ Total } & $\begin{array}{c}26 \% \\
(n=52)\end{array}$ & $\begin{array}{c}59 \% \\
(n=118)\end{array}$ & $\begin{array}{c}8,5 \% \\
(n=17)\end{array}$ & $\begin{array}{l}1,0 \% \\
(n=2)\end{array}$ & $\begin{array}{l}1,0 \% \\
(n=2)\end{array}$ & $\begin{array}{l}4,5 \% \\
(n=9)\end{array}$ & $\begin{array}{c}100 \% \\
(n=200)\end{array}$ \\
\hline
\end{tabular}

Na tabela 1 a correlação foi significativa, comprovada por $p=0,000$; quando as mães que realizaram prénatal $95,5 \%$ (191) foram questionadas sobre a qualidade do atendimento $59 \%(n=118)$ afirmaram ser boa, 26\% $(n=52)$ disseram muito boa, 8,5\% $(n=17)$ relataram satisfatória, $1 \% \quad(n=2)$ acharam ruim e 1\% $(n=2)$ consideraram muito ruim, já para as mães que não participaram das consultas de pré-natal 4,5\% ( $n=9)$ essa correlação não se aplica.

Na pesquisa, também foi encontrada uma correlação existente entre frequência com que o profissional responsável pela consulta do pré-natal realizava a aferição da pressão arterial das mesmas, e 94,5\% ( $n=181$ ) afirmaram ter ocorrido em todos os atendimentos, $5 \%(n=9)$ informaram ser em algumas consultas e 0,5\% ( $n=1)$ disseram não ter sido realizado em nenhuma. Quando indagadas sobre a aferição do peso, 97\% ( $n=185$ ) garantiram ter sido visto em todas as consultas e $3 \%(n=6)$ disseram ter feito em algumas consultas, mostrando a realidade do atendimento nos serviços procurados, principalmente nas unidades básicas e o compromisso dos profissionais em realizar um atendimento adequado para toda gestante. Todas apresentaram um $p=0,00$.

Outro questionamento foi se as gestantes realizaram alguns dos exames preconizados pelo pré-natal, percebendo-se que 98\% ( $n=188)$ realizaram Ultrassonografia, 95\% ( $n=181)$ fez exame de anemia e de urina, 94\% ( $n=180)$ submeteram ao teste de HIV, 88\% ( $n=168)$ realizou o de glicemia, 48\% ( $n=91)$ fez o teste de sífilis, ou seja, os profissionais sabem a real importância da realização desses exames junto com uma avaliação clínica para conduzir uma assistência eficaz às grávidas e seu filho, revelando uma correlação positiva comprovada pelo $p=0,00$. 


\section{ciência \\ plural}

Tabela 2: Correlação entre realização do pré-natal e complicações na gestação.

Santa Cruz/RN, Brasil, 2014.

\begin{tabular}{cccccc}
\hline & \multicolumn{5}{c}{ COMPLICAÇÕES } \\
\hline
\end{tabular}

De acordo com a tabela 2, foi calculada a Odds Ratio das participantes que fizeram pré-natal e daquelas que não fizeram pré-natal, de modo que foi achada uma prevalência de 0,86 , ou seja, $86 \%$ das mães que não complicaram estando presentes nas consultas de pré-natal, ao passo que ainda analisando aquelas que fizeram, foi encontrado uma prevalência de 0,13 (13\%) que tiveram complicação na gestação, considerando algumas causas como: 9,4\% $(n=18)$ tiveram sangramento, 3,1\% $(n=6)$ convulsionaram, 2,1\% ( $n=4)$ infecção urinária, 1,6\% $(n=3)$ diabetes, $1 \%(n=2)$ hipertensão arterial e por fim 8,4\% $(n=16)$ que foi por causa desconhecida.

Avaliando aquelas que não fizeram pré-natal 4,5\% (n=9) foi percebido que 0,88 (88\%) não complicaram, e apenas 11\% complicaram. Assim, pode-ser verificar que as situações de complicações perpassam pelas questões multifatoriais, de modo que necessita de atuação profissional e maior rastreamento clínico e epidemiológico.

\section{Discussão}

Nesse estudo que avalia a correlação entre a adesão das gestantes nas consultas de pré-natal e complicações na saúde materno-infantil, mostra uma forte procura dessas usuárias por esse serviço na Unidade Básica de Saúde, e ainda retrata que essa assistência é de boa qualidade, refletindo na satisfação da mãe e familiares e consequentemente prevenindo eventos indesejáveis no decorrer da gestação, parto e puerpério.

O Gráfico 1 apresenta em que mês gestacional as mães procuraram um serviço de saúde para realizarem seu acompanhamento durante a gestação, sendo visto que a maior parcela dessa população realizou sua primeira consulta até o primeiro trimestre de gravidez, manifestando atenção e interesse nessa nova etapa da vida. 


\section{ciência plural}

De acordo com outros estudos, na maioria das vezes a inadequação da participação do pré-natal esta associado a vários fatores sugestivos da desigualdade social, revelando que os indivíduos socialmente mais vulneráveis recebem uma assistência falha, demonstrando a lei da inversão do cuidado a ser prestado, onde os recursos para atenção à saúde são distribuídos contrariamente às necessidades. Assim é aconselhável o uso de táticas intervencionistas direcionadas aos grupos que necessitam de maior cuidado, no intuito não só de aumentar o número de gestantes em acompanhamento pela rede de serviços de saúde, como também a sua presença no serviço, envolvendo a gestante o mais cedo possível para o início da assistência pré-natal4.

De tal maneira, é de grande importância que o serviço que as usuárias procuram seja de fácil acesso, como também, que os profissionais de saúde estejam aptos a realizarem um atendimento humanizado e de qualidade para a gestante. Consequentemente a mesma retornará às consultas subsequentes e obterá resultados eficazes na sua saúde e de seu feto.

Assim, os profissionais do serviço devem ter alguns princípios fundamentais, tais como ser hábil e ser fonte de soluções para uma população estabelecida. Dessa forma, a afinidade equipe-paciente é alvo central na saúde da família e é competência também da equipe multiprofissional a receptividade e o acolhimento aos usuários, em especial as gestantes. Desse modo torna-se necessária a integração entre equipe e a comunidade no estabelecimento das preferências para o desenvolvimento do trabalho, tendo como referência o perfil epidemiológico da população adscrita, auxiliando no planejamento das ações 5 .

Dessa forma, a unidade de saúde deverá estar completa em seu quadro de profissionais pelos funcionários de nível médio e superior, pois é necessária a formação de uma equipe multiprofissional responsável e competente com o serviço a ser prestado, devendo possuir o mesmo objetivo que é de assistir os usuários de acordo com as suas necessidades e peculiaridades.

Contudo a participação no pré-natal deve iniciar imediatamente após o resultado positivo de gravidez, ter cobertura universal, ser marcado periodicamente, efetuar um número mínimo de seis consultas e o profissional de saúde precisa realizar suas ações de forma preventiva e curativa. Há alguns fatores essenciais para que a assistência seja um sucesso, que é a partir de que período se iniciou o acompanhamento, o número de atendimentos efetuados e os riscos à saúde que a gestante e seu concepto vivenciaram ${ }^{6}$. 


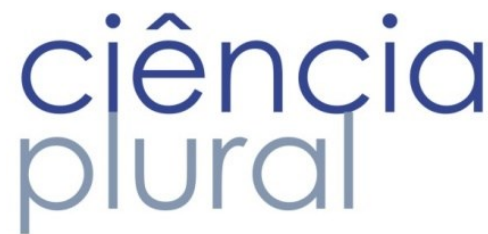

Assim a Estratégia de Saúde da Família e seus programas foram criados, no intuito de subsidiar a saúde de uma determinada população, com ações principalmente preventivas, de tal modo, que o pré-natal seja uma assistência prestada por toda a equipe de profissionais da unidade, desde os de nível médio até o de nível superior, pois cada um tem sua contribuição nessas ações, começando na busca ativa dos usuários até o atendimento ambulatorial resolutivo e de qualidade.

Por fim, a equipe de saúde da Estratégia de Saúde da Família tem o compromisso de promover a saúde, com ações baseadas não só em dados epidemiológicas e sanitários, mas considerando o contexto biopsicossocial do individuo, refletindo assim no aumento da qualidade de vida da sociedade. A promoção à saúde é alcançada quando a equipe presta assistência integral, efetiva, contínua e com qualidade, considerando 0 aspecto da família, com abordagem interdisciplinar, planejamento de ações, organização do trabalho e compartilhando decisões a serem tomadas. Dessa forma o usuário conseguirá resolver seus problemas e sentir-se satisfeito, confiando nos profissionais e admitindo maior envolvimento e participação nas intervenções?

No entanto, a ESF ainda está em processo de aperfeiçoamento, onde há muitas questões a serem solucionadas, porém para que isso seja possível é necessário que haja empenho e a participação ativa dos gestores, profissionais e usuários do serviço em prol de uma assistência qualificada direcionada a prevenir e solucionar os agravos existentes na sua região.

A tabela 1 mostra o que as genitoras que participaram do pré-natal consideram sobre a assistência prestada, onde a maioria afirmou ter sido boa e em seguida de muito boa, sendo esse um quesito muito importante para a assiduidade das mesmas no decorrer das consultas, como também para a adesão de futuras genitoras à esse serviço.

Estudos relatam que os procedimentos realizados no pré-natal são apontados como os melhores resultados de adequação, onde essas ações não exigem uma estrutura sofisticada de atendimento e necessitam quase que, unicamente, do desempenho dos recursos humanos. Isso confirma que os profissionais envolvidos na atenção do pré-natal valorizam a prática desses procedimentos e compreendem sua importância para a qualidade da assistência ${ }^{7,8}$.

Assim sendo, a gestação é considerada um período ímpar na vida de uma mulher, onde o ideal é que esse momento ocorra de forma saudável, havendo as alterações fisiológicas normalmente sem fornecer nenhum risco à mãe e a seu concepto, necessitando assim de um cuidado de qualidade à saúde dos mesmos. 


\section{ciência \\ plural}

Portanto, o risco de desistência ou de diminuir a frequência às consultas, por parte das genitoras, aumenta quando os profissionais de saúde não formam laços com a gestante ou não tratam essa questão como relevante, pois as mesmas sempre procuram aquele serviço que the passe maior segurança, em busca de boas condições para realizar o acompanhamento adequado do desenvolvimento e da saúde de ambos 9 .

Dessa maneira é necessário que haja compromisso e responsabilidade dos profissionais para com essas gestantes, sempre as mantendo informada do estado de saúde que se encontram, confirmar as atividades disponíveis à elas na unidade e demonstrar interesse no bem-estar da mãe e seu filho.

Assim, foi visto em estudos que o principal fator de risco para a natimortalidade é a frequência ao prénatal menor que cinco consultas, sendo crucial para a prevenção de intercorrências a extensão da atenção prénatal, fazendo com que as gestantes recebam uma atenção mais adequada, tanto em relação ao número de consultas como em conteúdo de atendimento9,10.

Dessa forma, torna-se indispensável à realização do número adequado de consultas e os exames básicos preconizados pelo Ministério da Saúde, pois assim é possível que o profissional de saúde realize um atendimento detalhado das condições de saúde da gestante e seu feto, sem deixar a desejar na assistência prestada.

A tabela 2 mostra a relação que a grande maioria das genitoras, que fizeram e as que não participaram do pré-natal, não tiveram agravos gestacionais, e as complicações surgiram na mesma proporção das que não realizaram e das que frequentaram as consultas, mostrando que o surgimento desses agravantes precisa de uma maior avaliação, e que muitas vezes não dependente somente da presença das mães no pré-natal. O que se pretende aqui é mostrar por meio da OR que as grávidas que fizeram pré-natal tiveram adesão superior por se tratar de um programa já institucionalizado na rotina na rede de atenção à saúde. Por outro lado, deve realizar busca ativa daquelas que não fizeram ou ainda não aderiram sob a prerrogativa de prevenção de maiores agravos materno-infantis, bem como um nascimento saudável e sem prováveis complicações.

Nesse contexto, em países desenvolvidos, os agravos perinatais estão relacionados principalmente a doenças de difícil prevenção como as más-formações congênitas, descolamento prematuro de placenta e os acidentes de cordão. Já em nações em desenvolvimento, como o Brasil, as principais complicações são a hipertensão gestacional, a infecção urinária e a sífilis congênita, onde são passiveis de prevenção por meio de adequada assistência pré-natal ${ }^{11}$. 


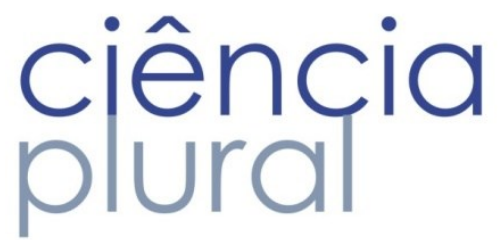

Um ponto relevante para um pré-natal direcionado é que os profissionais de saúde tenham conhecimento dos principais problemas da região, o que permite que possam adotar medidas que amenizem as repercussões indesejáveis na gravidez e, principalmente, saibam quais ações preventivas seriam mais eficazes à esse grupo de gestantes ${ }^{12}$.

Um dos fatores de risco importante para complicações materno-fetais é a obesidade, sendo hoje avaliada como um problema de saúde pública em mulheres em idade reprodutiva, onde pode aumentar o número de complicações obstétricas e neonatais, como 0 aumento dos partos cirúrgicos, prematuridade e recém-nascidos com peso alterado, assim como a hipertensão e eclampsia na gestação têm sido grandes causas de mortalidade materna em nossa população, e sem esquecer dos fatores familiares, sociodemográficos e econômicos que também podem colaborar com a manifestação de doenças referentes ao ciclo gravídico-puerperal13.

Outra questão importante a ser realizada é a identificação de fatores de risco que levam a mortalidade materna e neonatal, pois assim é possível subsidiar o plano de ações para a reestruturação e melhoria da atenção à gestante e aos recém-nascidos, visando à diminuição dos óbitos materno-infantil, que muitas das vezes não necessita de novos estudos, mas sim da garantia da acessibilidade e do uso mais eficaz do conhecimento científico e tecnológico existente ${ }^{14}$.

Nesse sentido é necessário que haja a educação continuada garantida pelos profissionais durante as consultas, mostrando as complicações mais prevalentes nas gestantes e como elas ocorrem, como também o modo de prevenir essas ações indesejáveis. Assim, será possível orientar e convencê-las do real sentido de obter uma vida saudável, para que possa dar continuidade à gestação com sucesso.

Outro ponto importante no pré-natal são os exames laboratoriais básicos, como de sangue, urina, fezes e ultrassom, pois eles ajudam na compreensão do que foi visto clinicamente, auxiliando no diagnóstico e na tomada de decisão em relação aos mesmos, mas foi visto que apenas metade das grávidas que são acompanhadas pelo serviço de saúde tem acesso a esses exames 6 .

Dessa maneira, é percebivel que quando o pré-natal não é realizado com a média de consultas exigidas e exames necessários, não é possível que o profissional de saúde estabeleça uma avaliação criteriosa do estado de saúde da mãe e de seu filho, podendo deixar muitos pontos importantes sem serem avaliados e possivelmente prejudicar a continuidade da assistência.

A realização das consultas periódicas, de exames como hemograma e ultrassom, costumes de vida saudável, como alimentação balanceada e a prática de exercícios físicos sem muitos esforços, é possível que 


\section{ciência plural}

tanto as mulheres que tem e as que não possuem predisposição a complicações gestacionais não as adquiram pelo hábito de vida.

Portanto, é indispensável uma maior atenção para as mães e filhos, sendo preciso a colaboração dos profissionais de saúde em proporcionarem uma atenção qualificada à esse público, como também dos gestores em nível municipal, estadual e federal a investirem na saúde oferecida à esses usuários, pois dessa forma é possível alcançar bons resultados na assistência prestada.

\section{Conclusões}

O pré-natal é a mais importante das assistências prestadas na unidade de saúde à mulher no decorrer da gestação, onde é possível realizar um acompanhamento saudável da gestante e seu filho, prevenindo e/ou tratando as complicações que podem surgir nesse período, concorrendo, assim, para a diminuição dos índices de morbimortalidade materna e infantil.

Dessa maneira, percebe-se a importância do profissional capacitado e compromissado com o serviço que o mesmo oferece, sempre atento às condições clínicas que as gestantes apresentam e orientando-as sobre a verdadeira necessidade de se obter uma vida saudável para dar seguimento à gestação sem intercorrências. É sob essa vertente que o Ministério da Saúde investe em cursos de capacitação e melhoria das condições assistências na Atenção Primária por entender a importância de um atendimento gratuito e de qualidade.

Outro fator de responsabilidade dos profissionais da assistência à saúde é a realização de um trabalho de busca ativa para com as gestantes que não aderiram ao pré-natal, conscientizando-as da importância da realização desse acompanhamento para a saúde materna e fetal, e ainda tornar o serviço resolutivo e de confiança aos olhos das futuras genitoras.

Há também questões que influenciam no surgimento de complicações nas gestantes que perpassa a assistência do pré-natal, como condições de moradia, renda familiar, educação das mulheres, urbanização, abastecimento de água e saneamento. Existem muitas ações governamentais que poderiam ser realizadas no intuito de melhorar as condições sociais da população, quais sejam: intensificar a melhoria na escolaridade da sociedade, a educação em saúde, o planejamento familiar, o pré-natal, a assistência hospitalar, com essas medidas é bem provável que reflita de forma positiva na redução das taxas de morbimortalidade materna e infantil do país.

De tal modo, ainda se faz necessário melhorar vários quesitos na assistência ofertada ao público, mas no âmbito da saúde, uma das maneiras é a realização de treinamentos e cursos às equipes de atendimento 


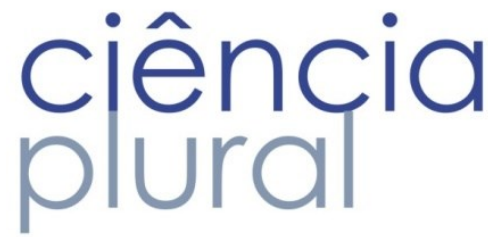

ambulatorial como também hospitalar, objetivando a qualificação destes, para que haja atitudes concretas e humanizadas desde o acolhimento até a resolução do problema.

Dessa forma, é possível realizar uma assistência voltada para prevenção, promoção e recuperação da saúde, baseada nos programas existentes na Estratégia de Saúde da Família, principalmente com o trabalho e dedicação de todos que fazem parte da equipe multiprofissional buscando garantir um atendimento digno, adequado, de qualidade humana e técnica para as mulheres que procuram a Unidade de Saúde em busca das consultas de pré-natal.

\section{Referências}

1. Assis TR, Viana FP, Rassi S. Estudo dos principais fatores de risco maternos nas síndromes hipertensivas da gestação. São Paulo: Arq. Bras. Cardiol [periódico online]. 2008. 91 (1). Disponível em: <http://www.scielo.br/scielo.php?script=sci_arttext\&pid=S0066-782X2008001300002\&lng=en\&nrm=iso> Acesso em: 21 mar. 2014.

2. Brasil. Ministério da Saúde. Secretaria de Vigilância em Saúde. Secretaria de Atenção à Saúde. Manual de vigilância do óbito infantil e fetal e do Comitê de Prevenção do Óbito Infantil e Fetal. Brasília [periódico online]. $2009 . \quad 96 \mathrm{p}$ Disponível em: <http://bvsms.saude.gov.br/bvs/publicacoes/manual_obito_infantil_fetal_2ed.pdf> Acesso em: 22 mar. 2014.

3. Monteiro CA, França Júnior I, Conde WL. Evolução da assistência materno-infantil na cidade de São Paulo. Rev Saúde Pública [periódico online]. 2000 []. 34: 19-25. Disponível em: <http://www.scielosp.org/pdf/rsp/v34n6s0/3515.pdf> Acesso em: 23 mar. 2014.

4. Coimbra LC, Silva AAM, Mochel EG, Alves MTSSB, Ribeiro VS, Aragão VMF et al. Fatores associados à inadequação do uso da assistência pré-natal. Rev. Saúde Pública [periódico online]. 2003]. 37 (4): 456-62. Disponível em: <http://www.scielo.br/pdf/rsp/v37n4/16780.pdf> Acesso em: 05 abr. 2014.

5. Duarte SJH, Andrade SMO. Assistência pré-natal no Programa Saúde da Família. Esc. Anna Nery [periódico online]. 2006. 10 (1): 121-25. Disponível em:<http://www.scielo.br/pdf/ean/v10n1/v10n1a16.pdf> Acesso em: 07 abr. 2014.

6. Grangeiro GR, Diogenes MAR, Moura ERF. Atenção Pré-Natal no Município de Quixadá-CE segundo indicadores de processo do SISPRENATAL. Rev. esc. enferm. USP [periódico online]. 2008. 42 (1): 105-111. Disponível: <http://www.scielo.br/pdf/reeusp/v42n1/14.pdf>. Acesso em: 10 abr. 2014.

7. Oliveira GS, Lima MCBM, Lyra CO, Oliveira AGRC, Ferreira MAF. Desigualdade espacial da mortalidade neonatal no Brasil: 2006 a 2010. Ciênc. saúde coletiva [periódico online]. 2013. 18 (8): 2431-41. Disponível em: <http://www.scielosp.org/pdf/csc/v18n8/28.pdf> Acesso em: 15 abr. 2014.

8. Koffman MD, Bonadio IC. Avaliação da atenção pré-natal em uma instituição filantrópica da cidade de São Paulo. Rev. Bras. Saude Mater. Infant. [periódico online]. 2005. 5 (1): 23-32. Disponível em: <http://www.scielo.br/pdf/rbsmi/v5s1/27838.pdf> Acesso em: 18 abr. 2014. 


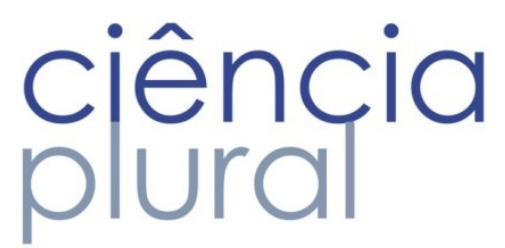

9. Almeida CAL, Tanaka OY. Perspectiva das mulheres na avaliação do Programa de Humanização do PréNatal e Nascimento. Rev. Saúde Pública [periódico online]. 2009. 43 (1): 98-104. Disponível em: http://www.scielo.br/pdf/rsp/v43n1/7296.pdf> Acesso em: 23 abr. 2014.

10. Rouquayrol MZ, Correia LL, Barbosa LMM, Xavier LGM, Oliveira JW, Fonseca W. Fatores de risco de natimortalidade em Fortaleza: um estudo de caso-controle. Jornal de Pediatria [periódico online]. 1996. 72(6). Disponível em: <http://www.jped.com.br/conteudo/96-72-06-374/port.pdf> Acesso em: 30 abr. 2014.

11. Silva EP, Lima RT, Ferreira NLS, Costa MJC. Pré-natal na atenção primária do município de João PessoaPB: caracterização de serviços e usuárias. Recife: Rev. Bras. Saúde Matern. Infant. [periódico online]. 2013. 13 (1): 29-37. Disponível em: <http://www.scielo.br/scielo.php?script=sci_pdf\&pid=S151938292013000100004\&lng=pt\&nrm=iso\&tlng=PT> Acesso em: 16 out. 2014.

12. Faria DGS, Zanetta DMT. Perfil de mães adolescentes de São José do Rio Preto/Brasil e cuidados na assistência pré-natal. Arq Ciênc Saúde [periódico online]. 2008. 17-23. Disponível em: <http://www.cienciasdasaude.famerp.br/racs_ol/vol-15-1/IIIIDDDD259.pdf> Acesso em: 10 set. 2014.

13. Santos EMF, Amorim LP, Costa OLN, Oliveira N, Guimarães AC. Perfil de risco gestacional e metabólico no serviço de pré-natal de maternidade pública do Nordeste do Brasil. Rev. Bras. Ginecol. Obstet. [periódico online]. 2012. 34 (3): 102-6. Disponível em: <http://dx.doi.org/10.1590/S0100-72032012000300002> Acesso em: 12 set. 2014.

14. Kassar SB,Melo AMC, Coutinho SB, Lima MC, Lira PIC. Fatores de risco para mortalidade neonatal, com especial atenção aos fatores assistenciais relacionados com os cuidados durante o período pré-natal, parto e história reprodutiva materna. J. Pediatr [periódico online]. 2013. 89 (3): 269-77. Disponível em: <http://www.scielo.br/pdf/jped/v89n3/v89n3a09.pdf> Acesso em: 15 out. 2014. 